\title{
NOISE DEDUCTION IN NOVEL PADDY DATA REPOSITORY USING FILTERING TECHNIQUES
}

\author{
MALATHI V. *AND GOPINATH M.P ${ }^{\dagger}$
}

\begin{abstract}
Classification of paddy crop diseases in prior knowledge is the current challenging task to evolve the economic growth of the country. In image processing techniques, the initial process is to eliminate the noise present in the dataset. Removing the noise leads to improvements in the quality of the image. Noise can be removed by applying filtering techniques. In this paper, a novel data repository created from different paddy areas in Vellore, which includes the following diseases, namely Bacteria Leaf Blight, Blast, Leaf Spot, Leaf Holder, Hispa and Healthy leaves. In the initial process, three kinds of noises, namely Salt and Pepper noise, Speckle noise, and Poisson noises, were removed using noise filtering techniques, namely Median and Wiener filter. The interpretation made over the median and Wiener filtering techniques concerning noises, the performance of the methods measured using metrics namely PSNR (peak to signal to noise ration), MSE (mean square error), Maxerr (Maximum squared error), L2rat (ratio of squared error). It is observed that the PSNR value of the hybrid approach is $18.42 \mathrm{~dB}$, which produces less error rate as compared with the traditional approach. Results suggest that the methods used in this paper are suitable for processing noise.
\end{abstract}

Key words: Salt and pepper noise; Speckle noise; Poisson noise; Median filter; Wiener filter.

AMS subject classifications. 60G35

1. Introduction. Agriculture is the primary resource to increase the economic growth of our country; it is the foundation of our economic framework. Paddy is the essential crops cultivated throughout the world, during the production period the majority of the yield loss occurs due to the diseases listed below Brown Spot, Bacterial Leaf Blight, and Blast. Diagnosing the diseases in an earlier stage using the computational approach plays an essential role in today's world [1]. Nowadays, the whole world depends on digital data, so processing the digital image is the most welcoming research topic. Data sets collected by camera are usually contaminated by noise. Noise is an essential factor that influences the quality of the image, which is primarily produced in the processes of image acquisition. Eliminating noise from the raw image is still a challenging issues for researchers. Thus, noise removal is the most necessary and the first step to be taken before the images is processed. It is essential to apply an efficient noise reduction technique to compensate for such data corruption. Image denoising remains a challenge for researchers because noise removal introduces artifacts and causes blurring of the images. So This paper mainly focuses and describes Wiener and median filtering techniques for noise reduction.

The combination of the pixel value forms an images. During image acquisition, there is a chance of changing the pixel value due to the noise present in the environment [2]. Noise might emerge during image capturing and transmission procedure, it corrupts the significant pixel value in the image, yet additionally influences the particular visualization of the image. Therefore, noise reduction plays a vital role in image processing and computer vision [3]. In Section 3, noises, namely Salt and Pepper noise, Speckle noise, Gaussian noise, and Poisson noise, are discussed. The above-listed noise is present in the digital image, and it is a tedious process to eliminate the specific noises in prior knowledge. The noise can be reduced either by linear or non-linear filter method; in noise filtering techniques, the most challenging task is to filter the noise without any information loss. Various kinds of noise reduction techniques, namely linear filter, min filter, max filter, median filter, wiener filter, Gaussian filter, guided, BM3D (Block matching and 3D filtering), and adaptive fuzzy switching median filter remove noises present in the image [4]. In the median filter, the performance can be enhanced by

\footnotetext{
${ }^{*}$ School of Computer Science and Engineering, Vellore Institute of Technology, Katpadi, Vellore, India.

$\dagger$ Associate Professor, School of Computer Science and Engineering, Vellore Institute of Technology, Katpadi, Vellore, India (mpgopinath@vit.ac.in).
} 
the researchers by utilizing the conventional median filter [5] [6] [7]. Dividing-conquering strategy improves the computational speed, and the complexity of the algorithm was achieved $O(N 2)$ to $O(n(\ln (n)))[8][9]$. In the proposed work, the novel data repository created and three noises, namely Salt and Pepper noise, Speckle noise, and Poisson noise, are introduced. Median and Weiner filtering techniques remove noises. The comparative study made with various kinds of noise concerning noise reduction techniques. Performance metrics, namely PSNR, MSE, Maxerr, L2rat used to evaluate the quality of the image.

The structure of the paper categorized as follows: Section 2 explains the related work; Section 3 describes the novel data repository and noise reduction techniques. Section 4 defines the strategies used to evaluate the quality of the images using various performance evaluation metrics. Section 5 report's the conclusion of the work.

2. Related Works. In this section, the recent contribution made by various researchers discussed below. Reza et al. [10] proposed a system to detect Jute Plant Leaf using Image Processing techniques and Machine learning techniques, the flow of the work categorize into two steps: initial stage involves pre-processing task, and the final stage is segmentation process. Bilateral filter techniques used to remove the noise present in the image and replaced with intensity value. Agarwal et al.[11] proposed denoising the images using various filtering techniques, namely special filter, wiener filter, and median filter. The comparative study conducted on these filtering techniques; at last, the author concluded that a wiener filter is a more suitable method to denoise the images. Orillo et al.[12] proposed a system using inverse laplacians; the issue of the monogenetic signal was handled efficiently by using this algorithm. Archana et al.[13] intended the detection of plant diseases, the essential investigating strategy for programmed recognizable proof is the sifting procedure of pre-processing technique. Henceforth, this Image filtering assumes a significant job to expel clamor from the image. Thus, this pre-processing strategy is the underlying stage to improve image quality. The comparison made over four different filters, namely Gaussian filter, median filter, mean filter, and Wiener by utilizing a standard data-set. The image nature of general outcomes shows that the examination of different separating strategy performed to upgrade quality. So, this paper gives the best beginning for specialists to the programmed discovery of rice plant sickness identification. The performance metrics evaluated using PSNR, SNR, and MSE. Through this work, among all the separating procedure, Weiner channel has preferred outcome over all the channels of most noteworthy PSNR qualities and SNR values. So these techniques used to pre-process dark-colored spot sickness in rice plants, which assists with dissecting further preparing.

Rani et al.[14] performed a comparative study of various filtering techniques. It was hard to state which filtering techniques are the best among all the filters. Exploring the outcomes by applying distinctive filter types to an image the accompanying resultants states: The Block Matching filter (BM3D) observed to be the stable method. The median filter is the best filtering technique for salt and pepper noise and Gaussian noise. Speckle noise works average for min and max filter, guided filter suits for Poisson noise. Salt and pepper noise can be removed effectively by using an Adaptive fuzzy median filter. At last, BM3D is a suitable method to eliminate all kinds of noises. Kaur [15] performed a comparative study over different filtering techniques by using the performance metrics. The performance of the filter varies, eventually concerning the noise present in the image. Salt and Pepper noise removed effectively using a median filter. Similarly, Poisson and speckle noise goes well with wiener filter. The essential results achieved recently by the authors in noise reduction techniques have mentioned in Table 2.1. The resultant analysis states that PSNR is the most frequently used noise measuring metrics and observed that the average PSNR value is around $23 \mathrm{~dB}$ for any kind of dataset, and a minimum of 14.45 can be achieved based on the kind of filtering techniques used.

\section{Proposed Work.}

\subsection{Novel Data Repository.}

3.1.1. Camera Specification. The images are captured with the help of high-resolution cameras, namely Canon EOS 1200D and FLIR E8 (Thermal camera). The specification of these cameras are mentioned below, Canon EOS 1200D 18MP, Digital SLR Camera (Black) with EF-S 18-55mm f/3.5-5.6, is II Lens and FLIR E8's crisp $76,800(320 \mathrm{X} 240)$ pixel infrared resolution, $+2 \%$ accuracy of reading for ambient temperature $10^{\circ} \mathrm{C}$ to $35^{\circ} \mathrm{C}\left(50^{\circ}\right.$ to $\left.95^{\circ} \mathrm{F}\right)$ and object temperature above $0^{\circ} \mathrm{C}\left(32^{\circ} \mathrm{F}\right)$, the field of view is $45^{\circ} \mathrm{X} 34^{\circ}$. 
TABLE 2.1

Review for Noise reduction using filtering techniques

\begin{tabular}{|c|c|c|c|c|c|c|c|}
\hline Year & author & methods & PSNR & SNR & SSIM & MSE & Dataset \\
\hline \multirow{3}{*}{2019} & \multirow{3}{*}{ Fan et al } & \multirow{3}{*}{ wiener filter } & 27.81 & - & 0.712 & - & set 12 dataset(lena images) \\
\hline & & & 22.95 & - & 0.647 & - & set 12 dataset(boat image) \\
\hline & & & 23.91 & - & - & - & set 12 dataset(monarch image) \\
\hline \multirow[t]{2}{*}{2018} & \multirow[t]{2}{*}{ Archana et al. } & Wiener filter & 38.2 & 14.98 & - & 42.75 & Paddy leaf \\
\hline & & Linear filter & 14.45 & - & - & 1330 & \\
\hline \multirow[t]{3}{*}{2018} & \multirow[t]{3}{*}{ Rani et al } & Min filter & 18.85 & - & - & 1075.52 & \\
\hline & & Max filter & 18.49 & - & - & 1156.6 & MATLAB cameraman image \\
\hline & & Median filter & 29.21 & - & - & 237.5 & \\
\hline \multirow[t]{2}{*}{2012} & \multirow[t]{2}{*}{ Zhu et al } & Wiener filter & 25.93 & - & - & 165.96 & Lena image \\
\hline & & Median filter & 32.118 & 26.105 & - & - & \\
\hline
\end{tabular}

3.1.2. Image Collection. The images collected from the state of Tamil Nadu include the following regions; the Agri field (VIT School of Agricultural Innovations And Advanced Learning. (VAIAL), VIT, Vellore), Brahmapuram, sevur, Latheri, vaduthangal from Vellore district. The complete field survey has undergone concerning disease symptoms and climatic conditions; samples were collected and captured using two different high-resolution cameras, as mentioned in section 3.1.1. Later, the diseases classified with the help of plant pathologists from VAIAL, Vellore Institute of Technology, Vellore. In the region, as mentioned above, the paddy crop leaves are affected by two significant reasons, namely Diseases and Pest. The leaves turn to yellow; the brown spot appears over the leaves; insects consume the leaves as well as hold the leaves and lay their eggs. Insect nest is the major problem in crops; the only solution to destroy the insect nest is clipping off the leaves, but this process can cause the bacteria to enter quickly through open pores and affects the plants. Different kinds of diseases include bacteria leaf blight, blight, leaf spot, leaf holder, hispa, and healthy leaves are collected in the region, as mentioned earlier, are illustrated in Table 3.1. Sample images of the data repository shown in Fig 3.1.

TABLE 3.1

count of the images included in the repository

\begin{tabular}{lllll}
\hline S.No & Diseases & $\begin{array}{l}\text { Number of images captured } \\
\text { using canon 1200D }\end{array}$ & $\begin{array}{l}\text { Number of images captured } \\
\text { using FLIR E8 }\end{array}$ & Total \\
\hline 1 & Bacteria leaf blight & 208 & 341 & 549 \\
2 & Blight & - & 206 & 206 \\
3 & Leaf spot & 188 & 225 & 413 \\
4 & Leaf holder & 54 & 69 & 123 \\
5 & Hispa & 53 & 317 & 370 \\
6 & Healthy leaves & - & 427 & 427 \\
\hline
\end{tabular}

3.2. Noise in Paddy images. The digital image categorized into binary, grayscale, and color images. In the binary image, the pixel value lies between zero or one (i.e., either black or white). In a grayscale image, pixel value lies between 0 to 255, and this implies every pixel right now appeared by eight bits, which actually of one byte. The color image generated by the combination of the red, green, and blue pixel values. Therefore 2563 different combination color values are obtained. During the image capturing process, due to the ultraviolet radiation and the dust present in the environment causes variation in the pixel value. Salt and pepper noise, Gaussian noise, speckle noise, and Poisson noise are the kinds of noises present in the image [15].

3.2.1. Salt and Pepper noise. Salt and pepper noise comes under the category of impulse; the resultant of this noise leads to white dot scattered over the image, and the pixel value is in dynamic range. The salt and 

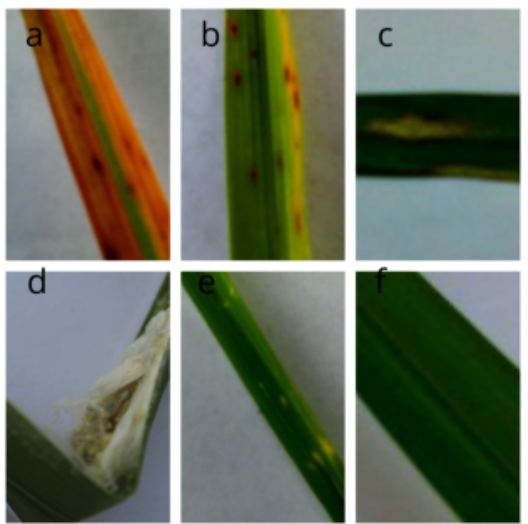

FIG. 3.1. Sample image data repository a. Bacteria leaf blight; b. Leaf spot; c. Blast; d. Leaf holder; e. Hispa; f. Healthy leaf

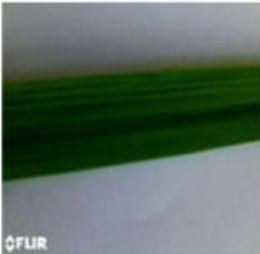

a

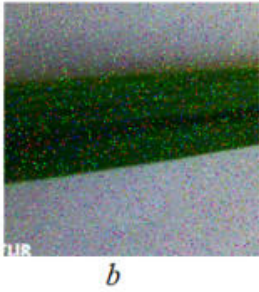

$b$

FIG. 3.2. a. Input image of healthy leaves; $b$. images with salt and pepper noise

pepper noise occurs due to the following issues like a malfunction in sensor, camera, information transmission, and storage allocation [16]. Fig 3.2 illustrates, salt and pepper noise added to the original image, i.e., salt noise induced by adding a brightness value of 255 pixels and pepper noise influenced by adding darkness with 0 -pixel values.

3.2.2. Poisson Noise. Poisson, commonly known as shot photon noise, occurred when the count of photons detected by the senor isn't adequate to give distinguishable statistical data. Shot clamor might be overwhelmed when the limited number of particles that convey vitality is adequately little, so vulnerabilities because of the Poisson dispersion, which depicts the event of autonomous irregular occasions, are of centrality [17]. Fig 3.3 illustrates the induction of Poisson noise in the bacteria leaf blight.

3.2.3. Speckle Noise. Speckle noise is commonly known as Multiplicative noise. The noise was created by multiplying random value concerning pixel value and illustrated by using formula (3.1) mentioned below. Speckle noise observed in a radar system, even though it might show up in a remotely detected picture using lucid radiation [18]. Fig 3.4 illustrates the induction of speckle-noise over bacteria leaf blight and healthy leaves.

$$
s=x+u * x
$$

where $s$ represents the speckle noise, $x$ is the input image, $u$ is the uniform noise image, $m$ is the mean and $v$ represent the variance.

3.3. Noise reduction techniques. The above-discussed noise can be removed by applying filtering techniques to the noisy data. The various noise filtering techniques are Linear filter, Median filter, Wiener filter, Gaussian filter, Kuan filter, Bilateral filter, Adaptive median filter, Adaptive wiener filter, Mean filter, Adaptive fuzzy, Guided filter, BM3D filter. In this section, two primary filtering techniques, namely Median and Wiener filter, were applied over the data-set, and performances measured. Wiener filter is optimized to identify the mean square error estimate of the original signal from a noisy measurement. Wiener filter is the frequently used 


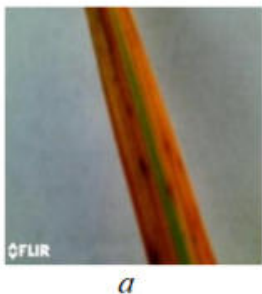

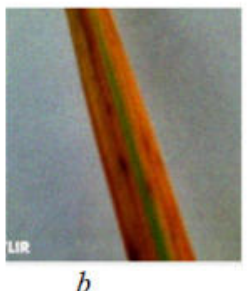

$b$

FIG. 3.3. a. Input image of Bacteria leaf blight disease; b.Images with Poisson noise

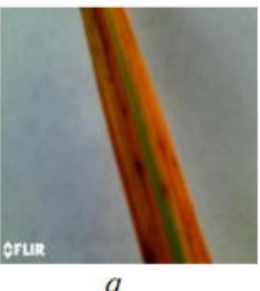

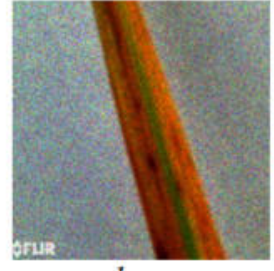

$b$

FIG. 3.4. a. Input image of Bacteria leaf blight disease; b.Images with speckle noise

technique to eliminate the blurriness present in the images. Wiener filtering is, in fact, the underlying premise for the restoration of other kinds of a blur; and being a least-mean-squares technique, it has roots in a spectrum of other engineering applications [20]. Mean filters usually remove the edges that have a high-frequency component, and this problem can overcome by using the median filter. The median filter protects the image information in horizontal, vertical, and diagonal directions. Computational complexity is low [21].

3.3.1. Median filter. Median filter falls under the non-linear filter type. In the median filtering technique, the smoothing method used to remove the noise present in the image. This filter furthermore brings down the intensity variety among one and the rest of a considerable number of pixels. The median is measured as follows, the pixel value arranged in ascending order concerning window size later, and the middle value replaced with the whole pixel value. The median filter measured using the formula (3.2) mentioned below [19].

$m(x, y)=\operatorname{medianw}(q, r), \quad$ where $(q, r) \in(x, y)$

Pros of the median filter are, it is simple to implement, no need for generating new pixel value and Less sensitive, so the noise pixel value are removed effectively [22].

3.3.2. Wiener filter. Wiener filter is the kind of statistical approach; Wiener filter design is to cut down the measure of noise display in a signal by examining the noiseless signal. It diminishes the bulk of noise present in the noise by a Statistical approach. Wiener filter produces better results as compared to linear filter, but it consumes more computational cost as compared with other filters, as mentioned in the previous session. The application of the Wiener filter such as, linear identification, channel restoration, equalization, and system detection. The pros and limitation of wiener filter are it uses a broad window side to do the smoothing process, and it is more suitable to reduce speckle noise present in the image. The obscure edge reduced by cutting down the Wiener size. Limitations of the Wiener filter are, computational cost is more. It doesn't support well for nature noise since the restoration process doesn't achieve perfection. In the Wiener filter, detecting power spectra is problematic [22].

\section{Results and Discussions.}

4.1. Software and hardware requirement. The experiment was implemented in the following hardware; Intel(R) Core(TM) i5-8300H CPU @ 2.30GHz, 8GB RAM, 64-bit Operating System, x64 based processor, and software specification; Matlab R2019b 64-bit. 
4.2. Performance evaluation metrics. The quality of the image measured using the performance evaluation metrics includes PSNR (peak signal-to-noise ratio), MSE (mean square error), Maxerr (maximum squared error), and L2rat (ratio of squared error).

4.2.1. PSNR. The term peak signal-to-noise ratio (PSNR) is an appearance for the ratio among the maximum probable rate (power) of a signal and the power of distorting noise that changes the quality of its illustration.

$$
\begin{gathered}
P S N R=20 \log 10 \frac{2 \alpha-1}{\sqrt{(M S E)}} \\
M S E=\frac{\|x-y\|}{N}
\end{gathered}
$$

4.2.2. MSE. MSE states the average error among raw images and the noisy image. The error represents the quantity of the raw image varies from the image

$$
M S E=\frac{1}{q p} \sum_{0}^{q-1} \sum_{0}^{p-1} m(i, j)-n(i, j)
$$

$$
[P S N R, M S E, M A X E R R, L 2 R A T]=\operatorname{measerr}(X, X A P P)
$$

Maxerr is the maximum absolute squared deviation of the data, $X$, from the approximation XAPP. L2RAT is the ratio of the squared norms of the signal or image approximation, $X A P P$, to the input signal or image $X$.

4.3. Interpretation over filtering techniques. The experiment analysis carried out using a different kinds of approaches. Initially, the three kinds of noises, namely Salt and Pepper noise, Poisson noise, and Speckle noise was induced over the dataset using imnoise function, later two different filtering techniques like Median filter and Wiener filter are applied to remove the noise as illustrated in Fig 4.1. Observation states that, for Salt and Pepper noise, the PSNR and MSE median filter is less than the wiener noise. So, concluded that the median filter works better as compared with the Wiener filter, as the resultant value illustrated in

\begin{tabular}{|c|c|c|c|c|c|c|c|c|c|c|}
\hline \multicolumn{11}{|c|}{ Type of filter } \\
\hline \multirow[t]{3}{*}{ Classes } & & & \multicolumn{4}{|c|}{ Median filter } & & \multicolumn{3}{|c|}{ Wiener filter } \\
\hline & PSNR & & MSE & MAXERR & L2RAT & PSNR & & MSE & MAXERR & L2RAT \\
\hline & Original & Denoise & & & & Original & Denoise & & & \\
\hline $\begin{array}{l}\text { Bacteria } \\
\text { leaf } \\
\text { blight }\end{array}$ & 37.2513 & 30.825 & 12.244 & 134 & 0.998 & 24.958 & 18.587 & 207.588 & 166 & 1.011 \\
\hline Blast & 35.986 & 28.640 & 16.384 & 170 & 0.997 & 23.015 & 15.746 & 324.719 & 227 & 1.0156 \\
\hline $\begin{array}{l}\text { Leaf } \\
\text { spot }\end{array}$ & 38.854 & 32.538 & 8.466 & 154 & 0.998 & 24.487 & 18.207 & 231.379 & 155 & 1.0070 \\
\hline $\begin{array}{l}\text { Leaf } \\
\text { holder }\end{array}$ & 37.888 & 31.63 & 10.573 & 153 & 0.999 & 24.852 & 18.637 & 212.738 & 188 & 1.0092 \\
\hline $\begin{array}{l}\text { Leaf } \\
\text { holder }\end{array}$ & 37.888 & 31.63 & 10.573 & 153 & 0.999 & 24.852 & 18.637 & 212.738 & 188 & 1.0092 \\
\hline Healthy & 38.603 & 32.268 & 8.968 & 143 & 0.998 & 24.200 & 17.905 & 247.17 & 201 & 1.0078 \\
\hline
\end{tabular}
Tables 4.1, 4.2 and 4.3.

TABLE 4.1

Performance evaluation metrics for salt and pepper noise, noises filtered using median and wiener filter. 
TABle 4.2

Performance evaluation metrics for speckle noise, noises filtered using median and Wiener filter.

\begin{tabular}{|c|c|c|c|c|c|c|c|c|c|c|}
\hline \multicolumn{11}{|c|}{ Type of filter } \\
\hline \multirow[t]{3}{*}{ Classes } & & & \multicolumn{4}{|c|}{ Median filter } & & \multicolumn{2}{|c|}{ Wiener filter } & \multirow{3}{*}{ L2RAT } \\
\hline & PSNR & & MSE & MAXERR & L2RAT & PSNR & & MSE & MAXERR & \\
\hline & Original & Denoise & & & & Original & Denoise & & & \\
\hline $\begin{array}{l}\text { Bacteria } \\
\text { leaf } \\
\text { blight }\end{array}$ & 29.7453 & 23.318 & 68.952 & 88 & 0.998 & 29.543 & 23.125 & 72.237 & 79 & 1.0004 \\
\hline Blast & 27.500 & 20.146 & 115.612 & 179 & 0.995 & 28.384 & 21.037 & 94.33 & 89.000 & 0.997 \\
\hline $\begin{array}{l}\text { Leaf } \\
\text { spot }\end{array}$ & 26.694 & 20.387 & 139.198 & 155 & 1.0008 & 28.956 & 22.661 & 82.68 & 77 & 1.0036 \\
\hline $\begin{array}{l}\text { Leaf } \\
\text { holder }\end{array}$ & 26.714 & 20.444 & 138.545 & 154 & 0.996 & 29.295 & 23.034 & 76.479 & 85 & 0.998 \\
\hline Hispa & 26.92 & 20.422 & 132.153 & 149.00 & 0.999 & 29.589 & 23.088 & 71.475 & 70 & 0.9999 \\
\hline Healthy & 26.802 & 20.453 & 135.784 & 139 & 0.995 & 29.461 & 23.125 & 73.613 & 75 & 0.998 \\
\hline
\end{tabular}

TABLE 4.3

Performance evaluation metrics for Poisson noise, noises filtered using median and Wiener filter.

\begin{tabular}{|c|c|c|c|c|c|c|c|c|c|c|}
\hline \multicolumn{11}{|c|}{ Type of filter } \\
\hline \multirow[t]{3}{*}{ Classes } & & & \multicolumn{4}{|c|}{ Median filter } & & \multicolumn{3}{|c|}{ Wiener filter } \\
\hline & PSNR & & MSE & MAXERR & L2RAT & PSNR & & MSE & MAXERR & L2RAT \\
\hline & Original & Denoise & & & & Original & Denoise & & & \\
\hline $\begin{array}{l}\text { Bacteria } \\
\text { leaf } \\
\text { blight }\end{array}$ & 34.594 & 28.159 & 22.576 & 127 & 0.996 & 37.298 & 30.8712 & 12.113 & 31 & 0.998 \\
\hline Blast & 34.499 & 27.137 & 23.076 & 177 & 0.9941 & 38.756 & 31.408 & 8.658 & 37.000 & 0.9974 \\
\hline $\begin{array}{l}\text { Leaf } \\
\text { spot }\end{array}$ & 35.421 & 29.094 & 18.659 & 159 & 0.996 & 37.501 & 31.185 & 11.558 & 30 & 0.998 \\
\hline $\begin{array}{l}\text { Leaf } \\
\text { holder }\end{array}$ & 34.72 & 28.455 & 21.928 & 150 & 0.997 & 37.341 & 31.077 & 11.992 & 30 & 0.998 \\
\hline Hispa & 34.797 & 28.291 & 21.543 & 143 & 0.998 & 37.512 & 31.005 & 11.529 & 25 & 0.997 \\
\hline Healthy & 35.248 & 28.906 & 19.419 & 141 & 0.997 & 38.043 & 31.711 & 10.203 & 28 & 0.999 \\
\hline
\end{tabular}

In the second approach since the kind of noises can't be easily detected in the data-set, so two different filtering techniques namely median and Wiener noise are applied over the data-set to remove salt and pepper noise, Poisson noise and wiener noise. The performance metrics illustrated in Table 4.4.

In the third approach, the hybrid technique followed, as illustrated in Fig 4.2, initially, introduce salt and pepper noise, Poisson noise, and speckle noise over the data-set. Later median filter techniques used to remove the noise. Then hybrid method (median and wiener filter) were used to remove the noise. The resultant value illustrated in Table 4.5. The observed value states that the error value is less and works better for the hybrid model. 


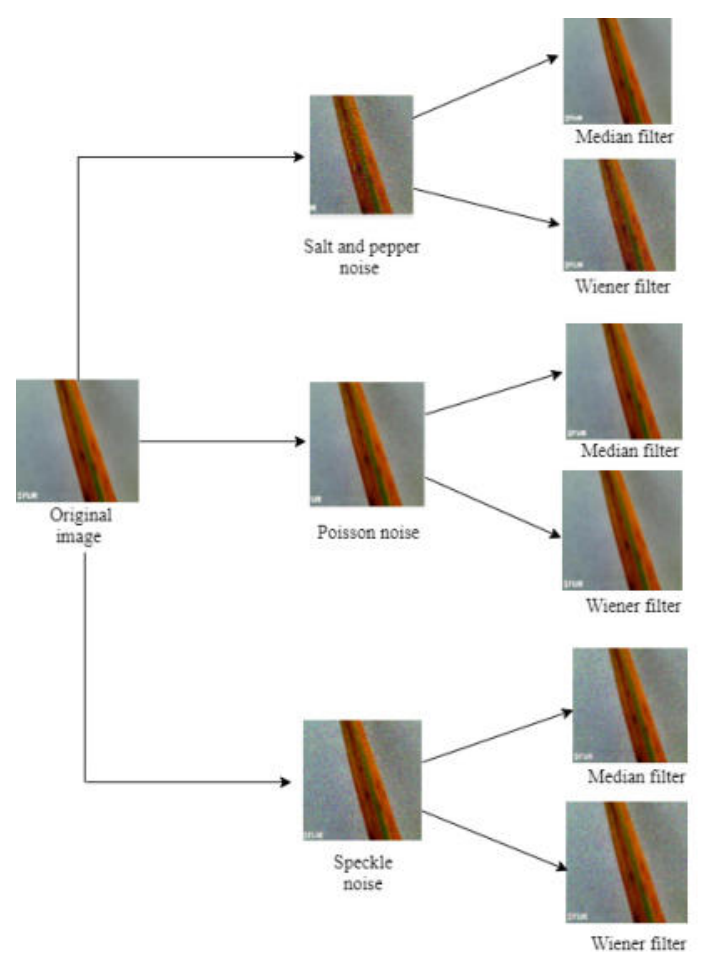

FIG. 4.1. Induced noises removed using median and Wiener filter

TABLE 4.4

Evaluation metrics for the given data repository using median and Wiener filter.

\begin{tabular}{|c|c|c|c|c|c|c|c|c|c|c|}
\hline \multicolumn{11}{|c|}{ Type of filter } \\
\hline \multirow[t]{3}{*}{ Classes } & & & \multicolumn{4}{|c|}{ Median filter } & & \multicolumn{3}{|c|}{ Wiener filter } \\
\hline & PSNR & & MSE & MAXERR & L2RAT & PSNR & & MSE & MAXERR & L2RAT \\
\hline & Original & Denoise & & & & Original & Denoise & & & \\
\hline $\begin{array}{l}\text { Bacteria } \\
\text { leaf } \\
\text { blight }\end{array}$ & 38.092 & 31.667 & 10.089 & 131 & 0.998 & 37.542 & 31.115 & 11.431 & 136 & 0.9984 \\
\hline Blast & 36.47 & 29.127 & 14.657 & 170 & 0.9985 & 36.244 & 28.897 & 15.439 & 175 & 0.997 \\
\hline $\begin{array}{l}\text { Leaf } \\
\text { spot }\end{array}$ & 39.24 & 32.523 & 7.745 & 144 & 0.9991 & 38.392 & 31.672 & 9.416 & 137 & 0.9985 \\
\hline $\begin{array}{l}\text { Leaf } \\
\text { holder }\end{array}$ & 38.262 & 32.0036 & 9.702 & 147 & 0.9992 & 37.675 & 31.416 & 11.105 & 144 & 0.998 \\
\hline Hispa & 38.2002 & 31.6987 & 9.842 & 139 & 0.991 & 37.655 & 31.152 & 11.156 & 140 & 0.998 \\
\hline Healthy & 38.913 & 32.581 & 8.3517 & 142 & 0.9993 & 38.606 & 32.27 & 8.963 & 142 & 0.9986 \\
\hline
\end{tabular}

5. Conclusion. In this work, image filtering techniques, namely Median filter and Wiener filter, were applied to remove the Salt and pepper, Speckle, and Poisson noises present in the given novel data repository. The interpretation made over various kinds of noise concerning noise reduction techniques. The quality of the image evaluated using performance metrics, namely PSNR (peak to signal to noise ration), MSE (mean square error), Maxerr (Maximum squared error), L2rat (ratio of squared error). The resultant value states that the median filter produces a better solution to eliminate noise as compared with a Wiener. In future work, we are planning to incorporate more filtering techniques over various kinds of crop leaves. 
TABLE 4.5

Hybrid approach.

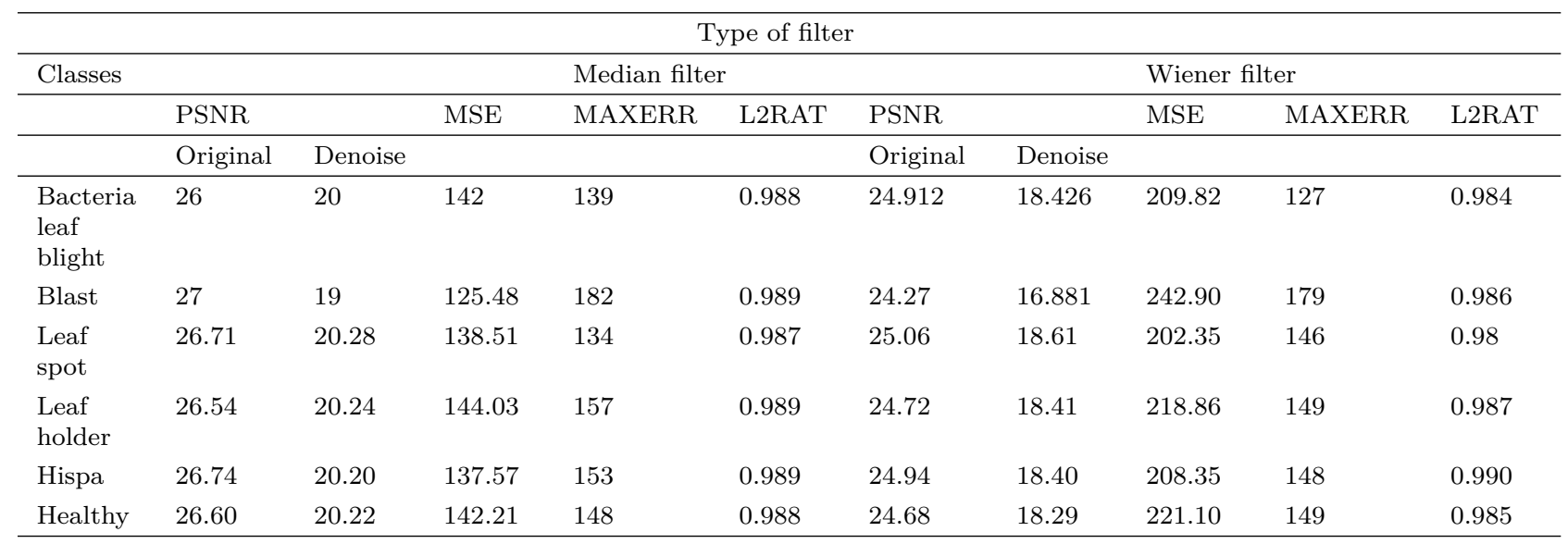

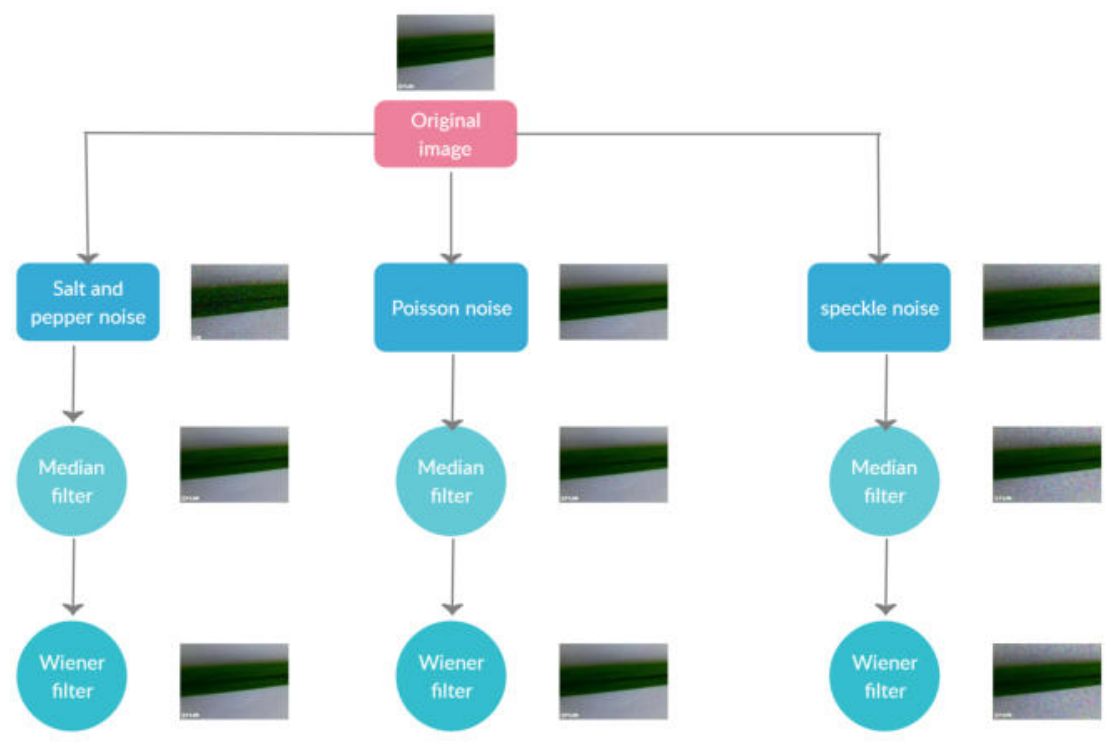

FIG. 4.2. A hybrid approach to reduce noise from the image data-repository

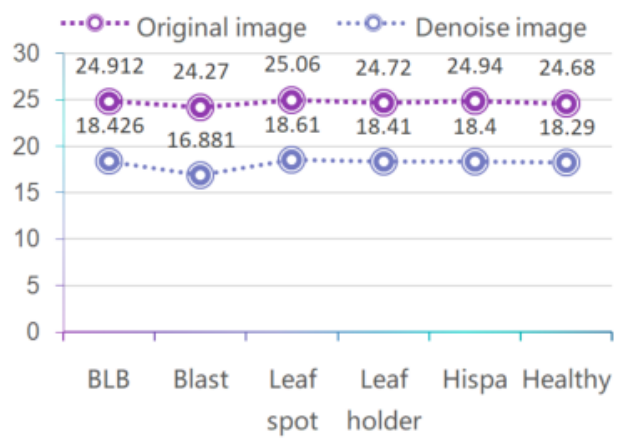

FIG. 4.3. Hybrid approach 
Acknowledgments. This research work was supported by Dr. Aarthy S.L and Dr. Sujatha R from the school of Information Technology and Engineering, Vellore Institute of Technology, and Dr. Priyadharshini B plant pathologist from VAIAL, Vellore Institute of Technology, Vellore.

\section{REFERENCES}

[1] Guchait N , Bhakta I. , Phadikar S. And Majumder K., Visual computing for blast and brown spot disease detection in rice leaves. , In proceedings of the 2 nd international conference on communication, devices, and computing, springer, singapore ,pp. 595-606, 2020.

[2] Rath A.K. And Meher J.K, Disease detection in infected plant leaf by computational method, Archives of phytopathology and plant protection, 1-11,2020.

[3] Chatterjee A., Roy S. And Das S.,Feature selection using rough set theory from infected rice plant images, In computational intelligence in pattern recognition, springer, singapore. Pp. 417-427, 2020.

[4] Brady, D. J., Hu, M., Wang, C., Yan, X., FAng, L., Zhu, Y. And Ma, Z , Smart cameras, Arxiv preprint arxiv:2002.04705, 2020.

[5] Guohong Liu, Wenming Guo,Application of Improved Arthmetic of Median Filtering Denoising,Computer engineering and applications, vol.46, no.10, pp.187-189,2010.

[6] Xiaokai Wang, Feng Li, Improved Adaptive Median Filtering ,Computer Engineering And Applications, vol.46, no. 3, pp. $175-176,2010$.

[7] Chao Wang, Zhongfu Ye, Salt-And-Pepper Noise Removal By Adaptive Median Filter And Tv , In painting, Journal Of University Of Science And Technology Of China, Vol.38, No. 3, Pp. 282-287, 2008.

[8] Qinghua Huang, Heqin Zhou, Huanqing Feng, A Fast And Effective Algorithm Of Pulse Noise Filtering For Imaging Data, Computer Engineering And Applications, No. 17, Pp. 113- 114, 210, 2002.

[9] T S Huang, G T Tang , A Fast Two- Dimensional Median Filtering Algorithm, IEEE Trans Acoustics, Speech, And Signal Processing, Vol.27, No. 1, Pp. 13- 18,1979.

[10] Reza Zn, Nuzhat F, Mahsa NA And Ali H , Detecting Jute Plant Disease Using Image Processing And Machine Learning, 3rd International Conference On Electrical Engineering And Information Communication Technology (ICEEICT),Pp. 16, 2016 .

[11] Agarwal SK and Kumar P, Denoising Of a Mixed Noise Color Image Using New Filter Technique, Proc.-Int. Conf. Comput. Intell. Commun. Networks, Pp.324-32, 2015.

[12] Orillo JW, Dela Cruz J, Agapito L, Satimbre PJ And Valenzuela I, Identification Of Diseases In Rice Plant (Oryza Sativa) Using Back Propagation Artificial Neural Network ,International Conference On Humanoid, Nanotechnology, Information Technology, Communication And Control, Environment And Management, 2014.

[13] Archana K S, And Sahayadhas A Comparison Of Various Filters For Noise Removal In Paddy Leaf Images ,International Journal Of Engineering and Technology, 7 2.21, 372-374, 2018.

[14] Rani, K. S., And Rao, D. N , A Comparative Study Of Various Noise Removal Techniques Using Filters , Journal Of Engineering And Technology, 7(2), 47-52, 2018.

[15] Kaur, S , Noise Types And Various Removal Techniques ,International Journal Of Advanced Research In Electronics And Communication Engineering (Ijarece), 4(2), 226-230, 2015.

[16] Halder, A., Sengupta, S., Bhattacharya, P., And Sarkar, A , Fast Adaptive Decision-Based Mean Filter For Removing Salt-And-Pepper Noise In Images ,In Computational Intelligence In Pattern Recognition, Springer, Singapore. Pp. 783-793, 2020 .

[17] Eckert, D., Vesal, S., Ritschl, L., Kappler, S., And Maier, A , Deep Learning-Based Denoising Of Mammographic Images Using Physics-Driven Data Augmentation, In Bildverarbeitungfür Die Medizin 2020, Springer Vieweg, Wiesbaden.(Pp. 94-100), 2020.

[18] Cruz, M. L, Full Image Reconstruction With Reduced Speckle Noise, From a Partially Illuminated Fresnel Hologram, Using a Structured Random Phase, Applied Optics, 58(8), 1917-1923, 2019.

[19] Das, S., Saikia, J., Das, S., And Goni, N, A Comparative Study Of Different Noise Filtering Techniques In Digital Images, International Journal Of Engineering Research And General Science, 3(5), 180-190. 2015.

[20] Diwakar M, Kumar M, A Review On Ct Image Noise And Its Denoising ,Biomed Signal Process Control 42:73-88, 2018.

[21] Jain P, Tyagi V , A Survey Of Edge-Preserving Image Denoising Methods ,Inf Syst Front 18(1):159-170, 2016.

[22] Zhu, Y., And Huang, C , An Improved Median Filtering Algorithm For Image Noise Reduction ,Physics Procedia, 25, 609-616, 2012.

[23] Fan, L., Zhang, F., Fan, H., And Zhang, C , Brief Review Of Image Denoising Techniques ,Visual Computing For Industry, Biomedicine, And Art, 2(1), 7, 2019.

Edited by: Rajkumar Rajasekaran

Received: Mar 27, 2020

Accepted: Aug 9, 2020 